\title{
Approach to Increasing the Carrying Capacity of the Pile Base
}

\author{
Sokolov NS* and IN Ulianov \\ Chuvash State University, Russia
}

*Corresponding author: Sokolov NS, Chuvash State University, Russia.

Received Date: July 13, 2020

Published Date: August 12, 2020

\section{Introduction}

The problem of increasing a foundation bearing capacity is always an urgent problem in geotechnical construction. With increased loads on the base, the use of traditional technologies is not always justified. It is necessary to use non-traditional bases strengthening methods. Most often, the situation is aggravated by the presence of weak underlying layers with unstable physical and mechanical characteristics in the engineering-geological sections. When reinforcing such bases with the help of traditional piles, they can get negative friction, reducing their bearing capacity by the ground. This article presents a developed algorithm for the construction of combined soil-concrete bored piles with simultaneous fixing of weak engineering and geological elements. At the same time, in areas with weak layers along the length of the piles, the developed geotechnical technology makes it possible to arrange broadenings obtained from the joint use of get-technology for the construction of soil-cement piles and the electric-discharge technology of bored electric discharge piles. The final result of the new technology is a soil-concrete pile with multi-seat broadenings, and which has an increased bearing capacity by the soil. In addition, in addition to the construction of deep reinforced concrete structures, in the presence of layers with reduced values of strength and increased deformation characteristics requires a special approach. Modern geotechnical construction in its arsenal has the methods and technologies to solve these complex problems. The use of advanced computer programs allows the development of geotechnical objects of any complexity. In order to identify the most acceptable geotechnical technology, the interactive design method must be used. This is "a developed project - an experimental site
- a real project." At the same time, this type of design should be multivariate using various geotechnical technologies and deep geotechnical reinforced concrete structures. Any stage in design must be economically sound and technically feasible. Often you have to deal with the geological conditions of construction sites, in the engineering and geological incisions of which there are overlapping layers with weak physical and mechanical indicators. The presence of such IEEs significantly reduces the carrying capacity of any piles on the ground, and sometimes leads to negative (negative) friction due to the overhang of the ground on the piles. It is known that foundations using drill-injecting piles have a number of competitive technical advantages over other foundations using other types of drilling piles. One of the distinctive parameters of some drilling piles from others is the technological possibility of including them in joint work with the surrounding soil. $1.1 \div 1.3$ (see table 7.6 pose 8 JV 24.13330.2011. Updated edition of SNIP 2.02.03-85 "Pile Foundations"). Thanks to the above, the increase in carrier capacity under the lower end of the piles can be 1.3 times or more, and on the side surface - exceed $1.1 / 0.5 \div 1.3 / 0.5$ q $2.2 \div 2.6$ times. In the event of a certain incomparability of Fdburrmule (7.11) CE 24.13330-2011, on 14.13330-2011, on 1999, the definition of $\mathrm{R}$ and $\mathrm{f}$ are determined on the basis of 7.3 and 7.8 CE 24.13330-2011. In the table. 7.3 CE 24.13330-2011 for the presentation of inland populations for railing of populations I LL and IL $\mathrm{f}$, ba in table. 7.8 CE 24.133302011 - then it is for R. For clarity, Veliing R / f = f (h) 1. Analyzing the table. 1 (see columns $4,7,13,16$ ) immediately catches the eye that the values of the $\mathrm{R} / \mathrm{f}$ ratio change significantly. $13,553,2$. This suggests that, with the help of special geotechnical 
measures, areas with weak layers can be replaced with a denser ground-cement structure called an intermediate support or a backto-air. At the same time, the size of the replacements expansions under the proposed geotechnical technology far exceeds the geometric dimensions of the expansions, In this case, in these areas with weak physicality co-mechanical indicators, the carrying capacity of the pile on the ground increases many Rd times. The author of this article has a lot of experience in the device of drills with intermediate supports. These are piles made by electrical discharge technology. With the help of electrohydraulic treatment Table 1: Dependencies $R / f=f(h)$ for various values of IL.

\begin{tabular}{|c|c|c|c|c|c|c|c|c|c|c|c|c|c|c|c|}
\hline \multirow[b]{2}{*}{1} & \multicolumn{3}{|c|}{$\mathrm{I}_{\mathrm{L}}-0.2$} & \multicolumn{3}{|c|}{$\mathrm{I}_{\mathrm{L}}-0.3$} & \multicolumn{3}{|c|}{$\mathrm{I}_{\mathrm{L}}-0.4$} & \multicolumn{3}{|c|}{$\mathrm{I}_{\mathrm{L}}-0.5$} & \multicolumn{3}{|c|}{$\mathrm{I}_{\mathrm{L}}-0.6$} \\
\hline & 2 & 3 & 4 & 5 & 6 & 7 & 8 & 9 & 10 & 11 & 12 & 13 & 14 & 15 & 16 \\
\hline $\mathrm{h}$ & $\mathrm{R}, \mathrm{kPa}$ & $\mathrm{f}, \mathrm{kPa}$ & $\mathrm{R} / \mathrm{f}$ & $\mathrm{R}, \mathrm{kPa}$ & $\mathrm{f}, \mathrm{kPa}$ & $R / f$ & $\mathrm{R}, \mathrm{kPa}$ & $\mathrm{f}, \mathrm{kPa}$ & $\mathrm{R} / \mathrm{f}$ & $\mathrm{R}, \mathrm{kPa}$ & $\mathrm{f}, \mathrm{kPa}$ & $\mathrm{R} / \mathrm{f}$ & $\mathrm{R}, \mathrm{kPa}$ & $\mathrm{f}, \mathrm{kPa}$ & $\mathrm{R} / \mathrm{f}$ \\
\hline 3 & 650 & 48 & 13,5 & 500 & 35 & 14,2 & 400 & 25 & 16,0 & 300 & 20 & 15,0 & 250 & 14 & 17,9 \\
\hline 5 & 750 & 56 & 13,7 & 650 & 40 & 16,3 & 500 & 29 & 17,2 & 400 & 24 & 16,7 & 350 & 17 & 20,6 \\
\hline 7 & 850 & 60 & 14,2 & 750 & 43 & 17,4 & 600 & 32 & 18,8 & 500 & 25 & 20,0 & 450 & 19 & 23,7 \\
\hline 10 & 1050 & 65 & 16,2 & 950 & 46 & 20,7 & 800 & 34 & 23,5 & 700 & 27 & 25,9 & 600 & 19 & 31,6 \\
\hline 12 & 1250 & 68 & 18,4 & 1100 & 48 & 22,9 & 950 & 36 & 26,4 & 800 & 28 & 28,6 & 700 & 19 & 36,5 \\
\hline 15 & 1500 & 72 & 20,8 & 1300 & 51 & 25,5 & 1100 & 38 & 28,9 & 1000 & 28 & 35,7 & 800 & 20 & 40 \\
\hline 18 & 1700 & 76 & 22,4 & 1500 & 53 & 28,3 & 1300 & 40 & 32,5 & 1150 & 29 & 39,7 & 950 & 20 & 47,5 \\
\hline 20 & 1900 & 79 & 24,1 & 1650 & 56 & 29,5 & 1450 & 41 & 25,4 & 1250 & 30 & 41,7 & 1050 & 20 & 52,5 \\
\hline 30 & 2600 & 81 & 32,0 & 2300 & 61 & 37,7 & 2000 & 44 & 44,0 & - & - & - & - & - & - \\
\hline$\geq 40$ & 3500 & 93 & 37,6 & 3000 & 66 & 45,4 & 2500 & 47 & 53,2 & - & - & - & - & - & - \\
\hline
\end{tabular}

We have also proposed strengthening the weak bases on the technology proposed in the "14" which is a synthesis of 3 geotechnical technologies:

1. Get-technology - the device of primer piles according to "JV 291.1325800.2017 Designs primed reinforced. Design rules. Moscow. 2017».

2. SFA technology is a device of drill-injecting piles with the of the ground of the walls of wells, it is possible to seal the sealed walls of drilling wells in the environment of fine-grained concrete over the river. As a result of this process, piles of ERT piles in the cross section in addition to the reinforced concrete section of the pile image are covered:

1) cement zone.

2) Seal area.

(Table 1) help of continuous passing screws (NPS) in the body of a primer array along its axis of symmetry.

3. The discharge-pulse technology of the driller pile device. The electro-hydraulic effect, which occurs when processing finegrained concrete, contributes to the introduction of it into the soiling array. This creates a more complete grip on these two design elements. 1 is a diagram of the device of the combined ground-concrete piles (Figure 1).

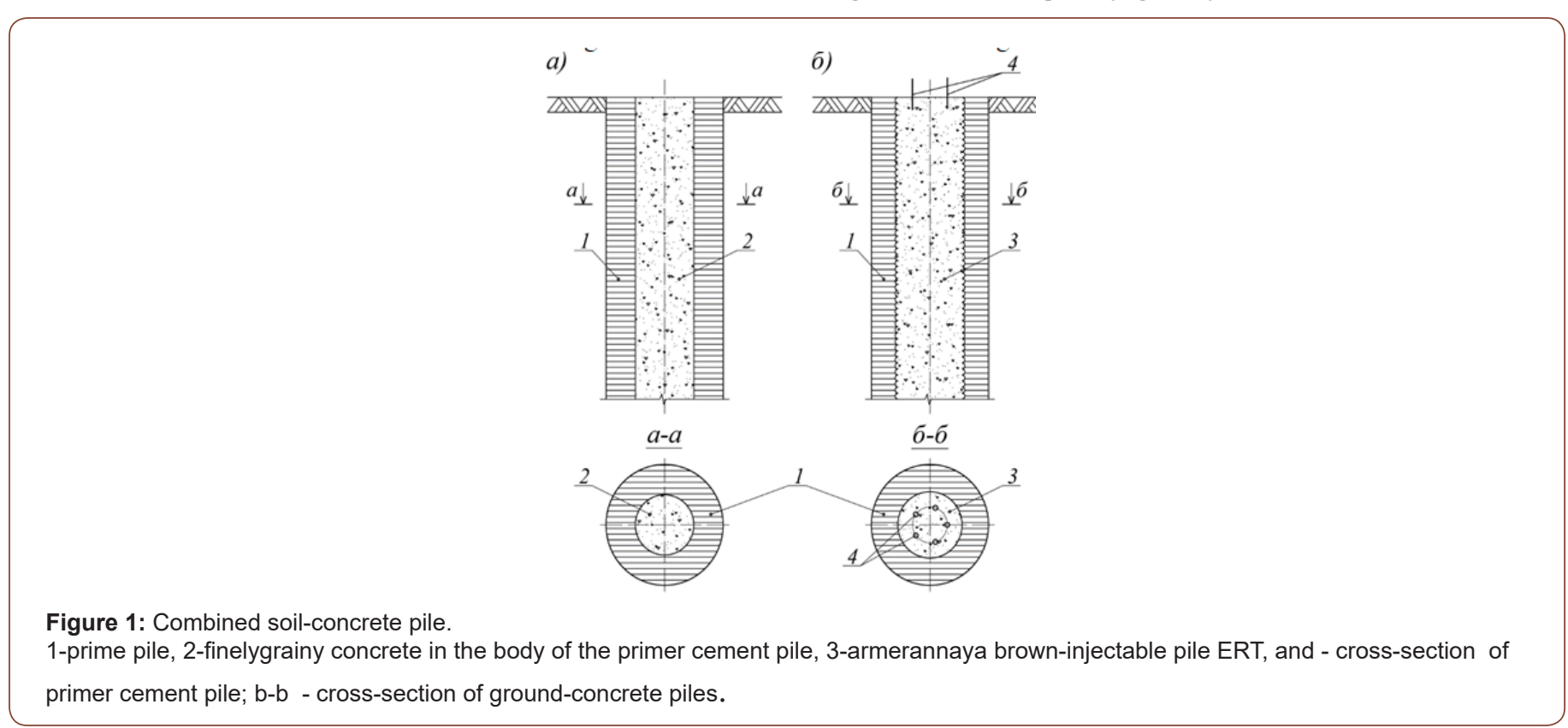


Deep reinforced concrete structure - ground-concrete pile (GWS) brought on rice. $1 \mathrm{~b}$ unlike other types has a complex crosssection design. The carrier is an electro hydraulically processed and reinforced SFA pile (POS) (pos. 3). The algorithm of the GBS device can easily be applied to the device of a dirt concrete piles with multi-local ears, as in a case of iron 2. (Figure 2).

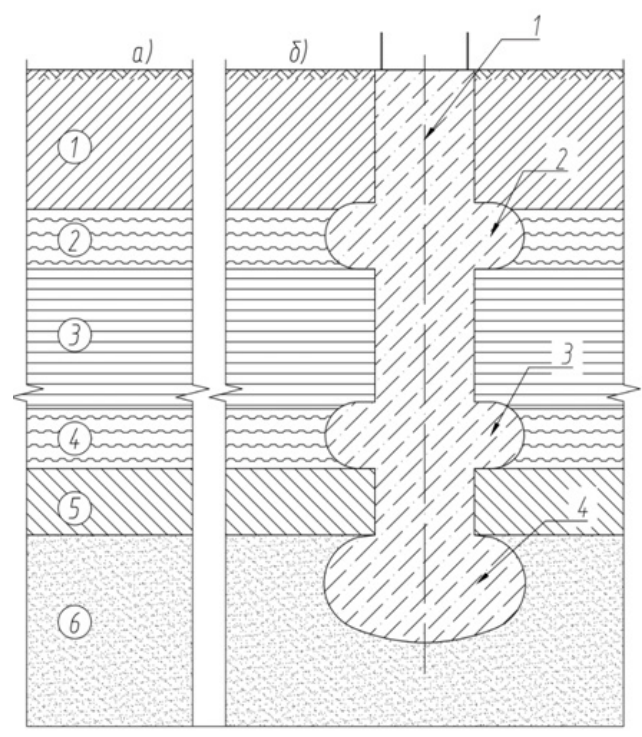

Figure 2: Inset in the engineerno-geological incision of the drilling ground-concrete piles with multi-local ears. (1) - (6) - engineeringgeological elements (IGE); (2), (4) - weak IHE; 1 - reinforced concrete trunk piles; 2, 3, 4 - broadening (glides).

In this Figure 2, the sinking's (under-heels) are arranged in IGE with weak physical and mechanical characteristics. Thus, we get a completely new geotechnical deep concrete structure, derived from the synthesis of two geotechnical technologies:

1. get-technology device cement piles;

2. Electro-discharge technology of the device of drill-injecting piles ERT.

At the same time, the newly created construction structure contributes to the achievement of increased values of the carrier capacity of the ground base.

\section{Acknowledgement}

None.

\section{Conflict of Interest}

No conflict of interest.

\section{References}

1. Ilyichev VA, Mangushev RA, Nikiforova NS (2012) Experience of the development of the underground space of Russian megacities. Grounds foundations and soil mechanics $2: 17-20$.

2. StreetVM,ShashkinAG, Shashkin KG(2010) Geotechnicalaccompaniment of urban development. St. Petersburg: Georeconstruction. 551

3. Ilichev VA (2004) Deformations of the Retaining Structures Upon Deep Excavations in Moscow. Ilyichev VA, Konovalov PA, Nikiforova NS, Bulgakov LA. Proc of Fifth Int Conf on Case Histories in Geotechnical Engineering New York 5-24.

4. Ilyichev VA (2007) Computing the evaluation of deformations of the buildings located near deep foundation tranches. Ilyichev VA, Nikiforova NS, Koreneva EB. Proc of the XVIth European conf on soil mechanics and geotechnical engineering Madrid Spain Geo-technical Engineering in urban Environments 2: 581-585.

5. Nikiforova NS (2011) Geotechnical cut-off diaphragms for built-up area protection in urban underground development. Nikiforova NS, Vnukov DA. The pros of the 7thI nt Symp Geotechnical aspects of underground construction in soft ground.

6. Nikiforova NS (2004) The use of cut off of different types as a protection measure for existing buildings at the nearby underground pipelines installation. Nikiforova NS, Vnukov DA. Proc of Int Geotech Conf dedicated to the Year of Russia in Kazakhstan Almaty Kazakhstan 338342 .

7. Petrukhin VP (2003) Effect of geotechnical work on settlement of surrounding buildings at underground construction. Petrukhin VP, Shuljatjev OA, Mozgacheva OA. Proceedings of the 13th European Conference on Soil Mechanics and Geotechnical Engineering, Prague.

8. Triantafyllidis Th (2007) Impact of diaphragm wall construction on the stress state in soft ground and serviceability of adjacent foundations. Triantafyllidis Th, Schafer R. Proceedings of the 14th European Conference on Soil Mechanics and Geotechnical Engineering Madrid Spain 683-688.

9. Sokolov NS (2018) Ground Ancher Produced by Elektric Discharge Technology, as Reinforsed Concrete Structure. Scopus Database Log -Key Enginiring Materials 76-81.

10. Sokolov NS (2018) Use of the Piles of Effective Type in Geotechnical Construction Scopus Database Log -Key Enginiring Materials. 70-74.

11. Sokolov NS (2018) One of Geotechnological Technologies for Ensuring the Stability of the Boiler of the Pit. Scopus Database Log-Key Enginiring Materials 56-69.

12. Sokolov NS (2018) Regulated injection pile-electric discharge technology with multiple pile enlargements posed as an underground reinforced concrete structure with a controlled load capacity. 18 international multidisciplenary scientific GeoConference SGEM 2018 Albena Resort SPA Bulgaria 601-608.

13. Sokolov NS (2018) One of the geotechnical technologies to strengthen the foundation base in constraint environment in the addition of 4 
floors. 18 international multidisciplenary scientific GeoConference SGEM Albena Resort SPA Bulgaria 513-522.

14. Sokolov NS, Viktorova SS (2018) Method of aliging the turches of objects targe-sized foundations and increased loads on them. Scopus Database Log -Key Enginiring Materials 1-11.

15. Sokolov NS, Sokolov AN, Sokolov SN, Glushkov VE, Glushkov AE (2017) Calculation of drill-injecting piles of increased carrying capacity. Housing construction 11: 20-26.

16. Sokolov NS (2017) High-carrying Foundation using drill-injecting piles of ERT with multi-local insibles. Housing construction 9: 25-29.

17. Sokolov NS, Viktorova SS (2017) Study and once the work of a discharge device for the manufacture of a booby-a-row - Construction: New technologies - New equipment. 12C: 38-43.

18. Nikolay Sokolov, Sergey Ezhov, Svetlana Ezhova (2017) Preserving the natural landscape on the construction site for sustainable ecosystem. Journal of applied engineering science 154(482): 518-523.

19. Sokolov NS (2018) Electropulse plant for the manufacture of drill piles. Housing construction 1(2): 62-66.
20. Sokolov NS (2018) One of the approaches to solving the problem of increasing the carrying capacity of drilling piles. Building materials 5: 44-47.

21. Sokolov NS (2017) Criteria for the economic efficiency of the use of drilling piles. Housing construction S: 34-38.

22. Sokolov NS, Sokolov SN, Sokolov AN, Fedorov P Yu (2017) And the use of drill-injecting piles of ERT as the foundations of the increased carrier capacity. Industrial and civil construction 9S: 66-70.

23. Sokolov NS (2019) Technology of increasing the bearing capacity of the base. Building materials 6S: 67-72.

24. Sokolov NS, Sokolov SN, Sokolov AN, Fedorov P Yu (2019) Drills - ERT as the foundations of the foundations of increased carrier capacity. Proceedings of the National Technical Conference with foreign participation Nonlinear mechanics of soils and numerical methods of calculations in geo-engineering and fundamental construction 195-201. 